\title{
25 Research Soure \\ An integrated design for collecting and recycling of space debris
}

Sherif Kamal ( $\square$ Dr.sherif.eeaa@gmail.com)

October 6 university https://orcid.org/0000-0002-6004-1977

Ahmed Gaber

Port Said University

Farouk El-Baz

Center for Remote Sensing, Boston University

\section{Letter}

Keywords: Space debris, Earth Orbit, Mitigation, Recycling, 3-D printing, ISS

Posted Date: July 1st, 2021

DOI: https://doi.org/10.21203/rs.3.rs-647907/v1

License: (9) This work is licensed under a Creative Commons Attribution 4.0 International License. Read

Full License 


\section{Abstract}

Today, as estimated by the U.S. Space Surveillance Network, there are 14,000 pieces of debris larger than 10 $\mathrm{cm}$ in Earth-orbit. That is in addition to thousands of satellites for various applications. As time passes, these satellites represent debris that might represent dangerous space waste. Such accumulated items orbit the Earth at very high speed and might threaten the daily uses of space-based applications, such as satellites that are needed for weather forecasting, telecommunications, and global positioning systems (GPS). In addition, this might negatively affect future missions - unless the international space community considers the risks and joins forces to mitigate them. Today, only ideas have been proposed by the various space agencies without taking much action. Each proposed idea deals with a specific size of debris. This paper proposes an integrated approach to collect most of the debris to be recycled for use as a source of raw material in construction equipment, which are used in maintenance of the International Space Station (ISS) and other equipment needed by future space missions and spacecraft. Furthermore, this proposal might solve the problem of the lack of space in the ISS, which served as home to numerous astronauts for more two decades. The proposed design could be implemented through cooperative programs by the world's space agencies. Because few countries represent the main source of this space wastes, it is here proposed that a financing source would be established by assigning a tariff for each future space flight, as a percentage of the cost of each mission. For example, fees could be determined based on; the extent of the mission's lifetime, the size and weight of the satellites, and the purpose of the mission.

\section{Background}

Each satellite in Earth orbit has an average lifetime around 5 years. In addition, many satellites have been converted to small debris with various sizes. Such orbital debris is called space waste. It is also defined as: "human-made objects in space that are no longer functioning because of decays, deorbits, explosions, or collisions of satellites, and thus, create more debris. This debris remains in orbit and rotates above the Earth's atmosphere. It survives for many years and might cause problems in the future, if the space community does not take any actions to deal with the potential risk.

The amount of the orbital debris has increased exponentially in the last few decades due to increase and accumulation of parts of satellites that were launched from many parts of the globe as well as unintentional or accidental spacecraft explosions and collisions. Collisions of larger debris create numerous fragments in the 1-kilogram mass range (David 2004). Orbital debris could destroy any satellite. Over 500,000 of pieces range from $10 \mathrm{~cm}$ to $1 \mathrm{~cm}$ size; being large enough to cause permanent damage. Moreover, more than 100 million particles range from $1 \mathrm{~cm}$ to $1 \mathrm{~mm}$ in size that could puncture a spacesuit or cause damage to fuel tanks and windows of space station and spacecraft. The hundreds of millions of space debris in Earth orbit were generated from the space activities of only a few countries. This includes more than 300,000 pieces of space debris large enough to completely destroy operating satellites upon impact (Wright 2007; Johnson 2009).

Thus, it is clear that Earth-orbital debris amplifies the risk of catastrophic collisions to spacecraft and a great danger to the crews. Moreover, the increasing volume of orbital debris threaten the important space-based applications, which are used in our daily life, such as weather forecasting, telecommunications, and global 
positioning systems (GPS) that are depend on orbiting satellites in a stable space environment (Paul K. Martin 2021).

We here propose an integrated design for a spacecraft that can be used to collect various sizes of space debris. The latter can be recycled for use as a source of raw material in construction equipment, maintenance of the International Space Station (ISS), or satisfying other needs of future space missions.

\section{Current Activities Of Monitoring And Tracking Space Debris}

To decrease space risks, there is a need to monitor, measure and track the debris in Earth orbit. The Orbital Debris Program Office (ODPO) at NASA has taken the national and international lead in conducting measurements of the orbital debris environment. It also has taken steps to develop the technical consensus for adopting mitigation measures to protect the space users. The ODPO collects its measurements from three ground-based radars (HUSIR, HAX, and Goldstone) and analyzes all the data through its modeling tools to depict the orbital debris environment. However, the operating time of these radars were limited due to lack of funding, inoperable equipment, and competing priorities of multiple users.

The two radars HUSIR and HAX were operated by the Massachusetts Institute of Technology's Lincoln Laboratory and utilized by both NASA and DOD. The HUSIR is the highest-resolution space-object-imaging radar in the world. It enabled it to statistically image the debris in its field-of-view and provide data about the size, shape, orientation, and motion of the objects. It also measures debris that ranges from $2 \mathrm{~cm}$ to as small as $5 \mathrm{~mm}$. The third radar that was utilized by ODPO is named Goldstone, and operated by the Jet Propulsion Laboratory. It is capable of detecting debris in the range of 3 to $5 \mathrm{~mm}$. This makes it a critical tool in the characterization of the sub-centimeter sized of space debris (Paul K. Martin 2021).

NASA cannot measure orbital debris below $3 \mathrm{~mm}$ in the most congested region (of the 600 to 1,000 km range) by using ground-based radars or telescopes. These particles are too small and too far away to be detected by ground-based radars such as HUSIR, HAX, and Goldstone, or through the U.S. Space Surveillance Network.

According to ODPO, direct measurement data obtained by space-based tools are needed to support the development of cost-effective protective measures for safe operation of future missions. Small debris items are extremely dangerous and can cause catastrophic damage to spacecraft due to the high collision speed. Such an item could travel at speeds of 17,500 miles per hour! That is fast enough to make small size items a serious safety concern for the over 400 space missions currently operating in Earth orbit (Mark 2013).

Millimeter-sized debris would also damage solar panels and communication arrays. During the Space Shuttle era, for example, several Shuttle windows had to be replaced because of damage caused by material that small size. Furthermore, such pieces may cause significant damage if it hits vulnerable parts of a satellite such as the fuel tank (Paul K. Martin 2021).

\section{Mitigation Efforts For Debris Removal}

Mitigation efforts and space debris removal become essential targets for a safe space environment. This became clear to everyone especially for existing operational satellites that are required for space-based 
applications used in our daily life. People everywhere became aware of that when they considered satellites in Earth orbit that rare needed for weather forecasting, telecommunications, and global positioning systems (GPS). All such knowledge is dependent on continued health of satellites in Earth orbit and also for the case of future missions in space. Thus, the world's space agencies are considering applicable solution to the collection of space debris.

NASA has funded programs to promote timely post-mission disposal of small spacecraft, as well as early stage studies of concepts for active debris removal. Therefore, NASA has developed the technology to demonstrate uncontrolled deorbiting of small spacecraft, such as CubeSats, using NASA's Exo-Brake Parachute that increases the spacecraft's drag on the braking device.

Although NASA has not developed an active debris removal system yet, the agency has been working on robotic refueling technologies that could provide additional fuel to extend missions and relocate or deorbit satellites (Paul K. Martin 2021). NASA has also developed a program to send the first 3-D printer to the space station in 2014. This printer used a fused filament fabrication (FFF) process, feeding a continuous thread of plastic through a heated extruder and onto a tray layer by layer to create a three-dimensional object. The 3-D printing in Zero $\mathrm{G}$ investigation produced dozens of parts, which researchers analyzed and compared with those made on the ground. Demonstrating that a 3-D printer works normally in space, this has paved the way to a new logistics system for long duration missions.

This project was designed to solve the problem of the lack of space in the ISS, which used to be the home of astronauts for more than nineteen years. Astronauts conduct scientific research using dozens of special facilities aboard the space station. The station also provides them with a place to eat, sleep, relax and exercise. To make all of this possible requires sending more than 7,000 pounds of spare parts to the station annually. Another 29,000 pounds of spaceflight hardware spares are stored aboard the station, and another 39,000 on the ground ready to fly if needed.

Thus, this article aims at utilizing recycled material for printer feedstock, in the Space Station which could save future long-duration exploration missions from having to carry a large supply of material for 3-D printing. Recycling also could make use of material that otherwise would represent a nuisance or a trash disposal issue on these missions.

In addition, the ISM project is working on developing higher-strength plastics and scaling processes that use metal to the size and power constraints of the Space Station. This is because most of the parts are need for on-demand manufacturing of spares - many are metal - on exploration missions, so that the program is studying the next big push in recycling metals using special 3-D printers (NASA 2019).

The European Space Agency's (ESA) debris removal mission known as ClearSpace-1, is slated to launch in 2025 (Fig. 1). It consists of a four-armed robot that would latch onto debris and descend back to earth, where both machine and debris will burn up upon entry into the atmosphere. The initial mission will target a piece of large debris an upper stage left over from a 2013 launch. In November 2020, the ESA announced that mission and cited that the remainder of the mission's cost would be raised through commercial investors (ESA 2019). 
Moreover, JAXA has introduced a debris removal mission in Japan. This one is designed to launch in two phases, an "approach and observe" phase in 2022 to 2023 and a follow-up "observe, approach, capture, and retrieve" phase in 2026. The intent is to collect a discarded large upper stage of a Japanese rocket selected for removal by JAXA (Fig. 2).

In addition, JAXA is working on a project to demonstrate the first ever "debris prevention" device in 2021. This new appraoach involves an electromagnetic tether mounted on a miniaturized satellite prior to launch. Then, at the end of the mission, it would extend into space, interact with the earth's magnetic field, and cause the satellite to slow. This results in the lowering of its orbit within a short period of time.This, in tern, results in burning of the satellite as it falls into Earth's atmosfere. (JAXA 2020).

\section{Proposed Space Debris Collection And Recycling System}

The collection and recycling sysytem proposed in this study, as shown in Figure 3, is designed for space debris to be used as a source of raw material. It is alsom assumed that it could be used for construction equipment for maintenance of the ISS.It can also be used for aequipment needed by the space missions as well as spacecraft of the future.

The proposed design for the collection and recycling sysytem directly helps the aim of the ISM program. The latter was introduced by NASA for use of 3-D printers in space to supply the needs of the ISS equipment. The latter were required for maintenance due to the lack of space in the ISS to store all spare parts. Therfore, the proposed design will help to decrease the number of missions required to send equipment and spare parts to the ISS.

Thus, the proposed design would save time, effort and funds required for future operations on future missions.

The proposed design is a hybrid system, It works in line with the goals and serves the) ClearSpace-1 (program of ESA and the JAXA use of an electromagnetic tether for space debris collection.

The proposed system can be divided into five stages, including:

Stage 1: Use of design similar to that of the ClearSpace-1 robot. It uses the 4 arm design by ESA in addition of introducing a flexable electromagnetic net between the robot arms. This is a similar technology to that used by JAXA to collect the space debris with diffrent sizes and types. At stage 1 the arms and the net are to be aligned in straight position as shown in the figure 4 toward the space debris cloud as controled by ground stations and the Internationl Space Station (ISS) crew.

Stage 2: Collection process, when the arms and the net extend as shown in the figure 5 to collect the largest amount of the space debris of varying size.

Stage 3: Closure of the robot arm and the flexable electromagnetic net to collect the space debris withen the net, and prevent any leakage of the collected debris as shown in the figure 6.

Stage 4: Moving the robot towards the ISS (not toword the earth as designed by JAXA or Clearspace-1 projects). During the journy a shredding and sorting machine is designed to render the debris into a suitable 
size to allow storage in the 3D printers of the ISS as shown in the figure 7.

Stage 5: Docking of the robot with the International Space Station as shown in the figure 8.

The robot works as a storage space for orbit debris after being shredded and sorted to be used as raw materials for the 3D printers (ISM program by NASA) to manifacture equipments and spare parts for the maintainence required for the ISS station and other long- time missions

\section{Conclusion}

It is extremely important to overcome the problem of Earth-orbital debris by collecting and removing it from space. This floating debris has negative and dangerous impacts. It represents a hazard to current operating satellites as well as future manned and unmanned missions. Therefore, there is an urgent need to introduce unconventional solutions and concentrate efforts by all space agencies. These agencies need to combine efforts and ideas to reach the most effective means and technologies to collect and recycle existing space debris.

This debris can be reused as source of raw material in manufacturing (using 3D printing technology), as well as for the production of spare parts for maintenance of space stations. This will save the time, effort and funds required for future operations and space missions. The three main European, Japanese and American agencies have programs for disposal or utilization of waste. Therefore, an integrated effort to merge these programs in one would lead to innovative and effective solutions.

The system proposed in this paper is to collect and integrate ideas by the major space agencies and fuse them into one effective effort. This would increase interest in solving the problem of space waste and decrease its negative impact on all future and current missions. In addition, this would help identify the best method for the collection and recycling of space debris to serve future goals in place of the simplistic thought of burning them up in the Earth atmosphere. Naturally, such a mega program requires funding. Thus, it is suggested that financing can be supplied through setting a tariff for each space mission, equal to from 3 to $5 \%$ of the cost of the mission. The collected funds can be used in research to result in solutions to the problem, and assure the recycling of space debris. This effort could also be used as a companion to those that seek the protection of the environment of the Earth.

\section{References}

- David O. Whitlock, “History of On-orbit Satellite Fragmentations, 13th Ed.”, NASA JSC 62530, May 2004.

- ESA commissions world's first space debris removal, 09/12/2019, http://www.esa.int/Safety_Security/Clean_Space/ESA_commissions_world_s_first_space_debris_removal.

- JAXA, June 5, 2020 ,https://global.jaxa.jp/press/2020/06/20200605-1_e.html

- Johnson, Nicholas L. 2009a. Statement before the House Subcommittee on Space and Aeronautics, Committee on Science and Technology, April 28 http://gop.science.house.gov/Media/hearings/space09/april28/johnson.pdf (accessed 27 November 2009). 
- Mark Garcia, Space Debris and Human Spacecraft Sep 27, 2013, https://www.nasa.gov/mission_pages/station/news/orbital_debris.html

- NASA, Solving the Challenges of Long Duration Space Flight with 3D Printing, Dec 16, 2019.https://www.nasa.gov/mission_pages/station/research/news/3d-printing-in-space-long-durationspaceflight-applications

- Paul K. Martin, NASA'S EFFORTS TO MITIGATE THE RISKS POSED BY ORBITAL DEBRIS, Report No. IG-21011, January 27, 2021, https://oig.nasa.gov/docs/IG-21-011.pdf

- Wright, David. 2009. Colliding Satellites: Consequences and Implications. Union of Concerned Scientists. http://www.ucsusa.org/assets/documents/nwgs/SatelliteCollision-2-12-09.pdf (accessed November 14, 2009).

\section{Figures}

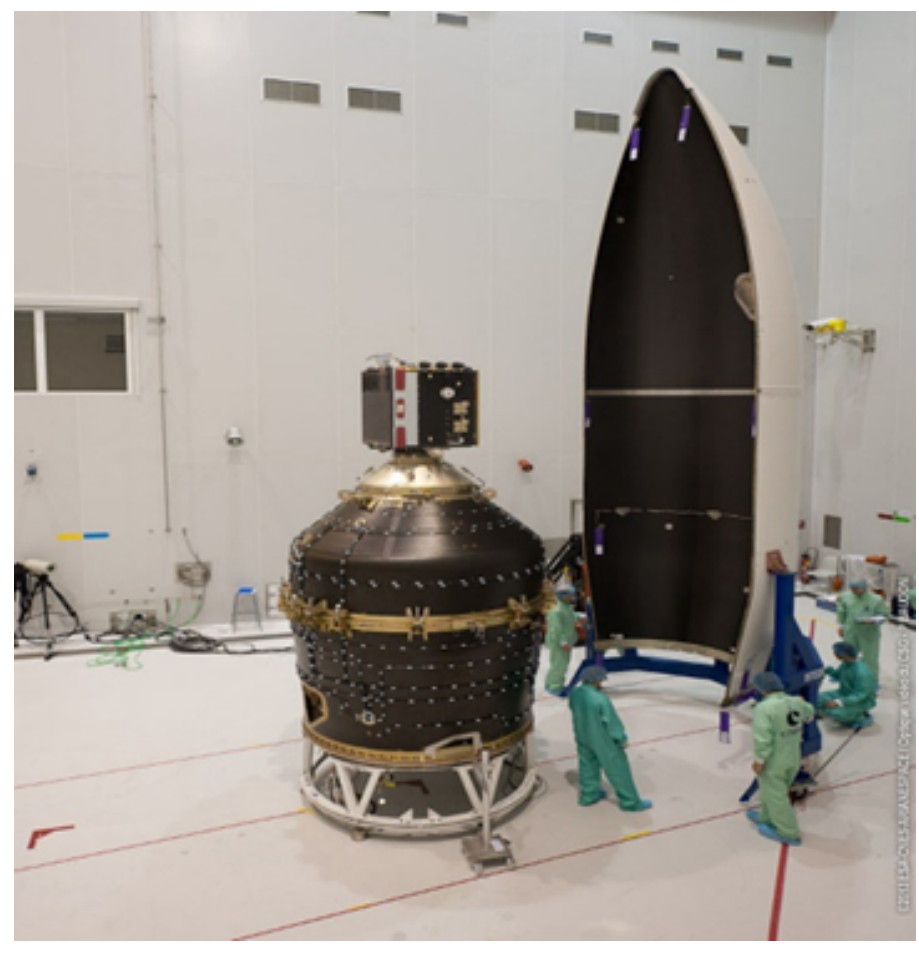

\section{Figure 1}

Clearspace-1 satellite (ESA 2019) 


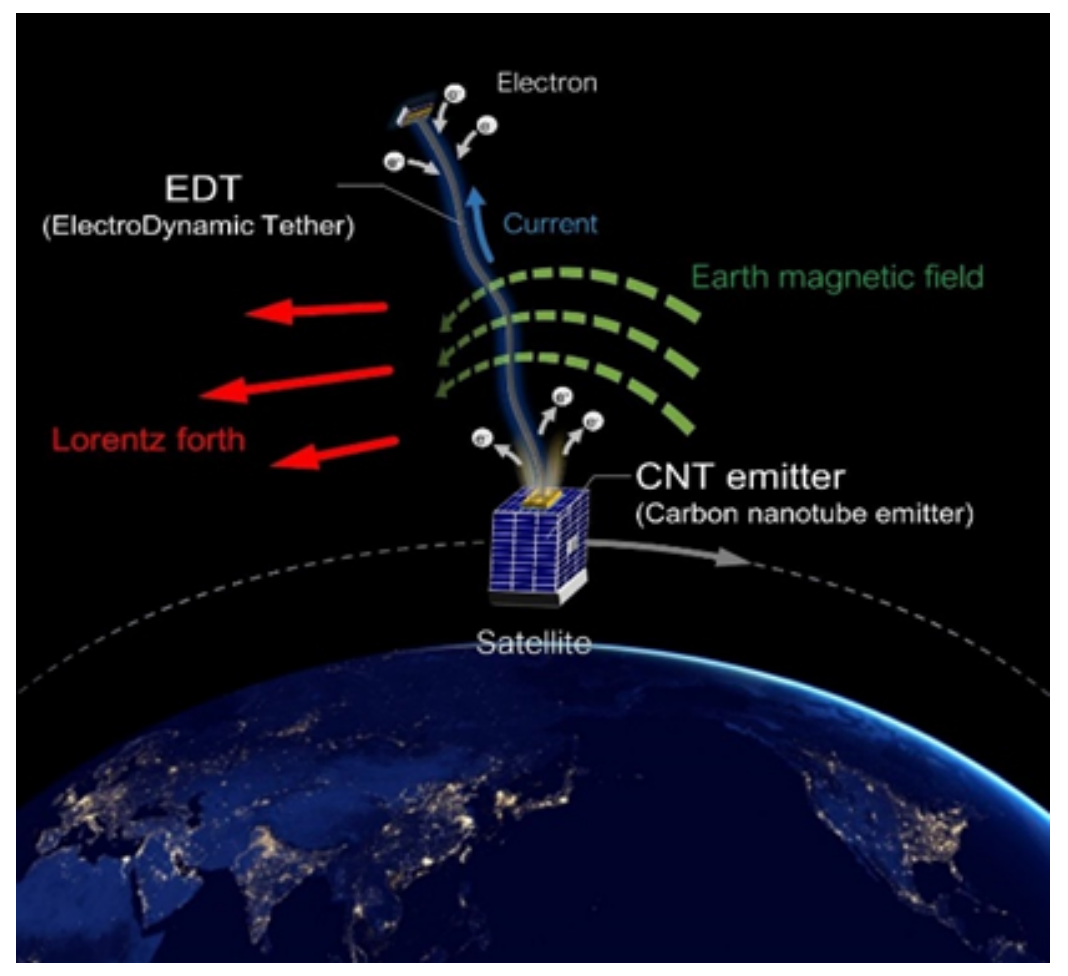

\section{Figure 2}

JAXA electromagnetic tether (JAXA 2020)

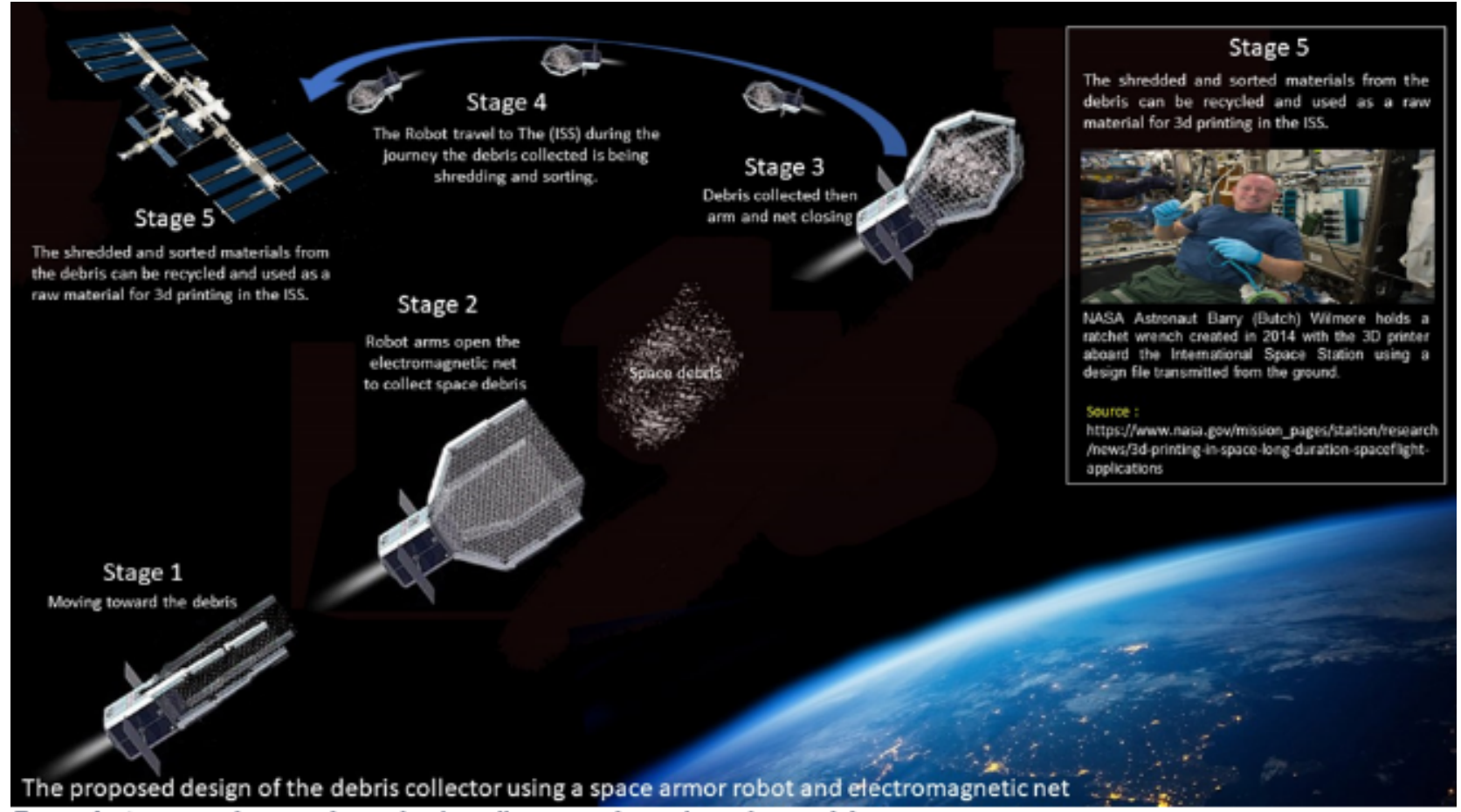

Figure $3:$ A proposed system design for the collection and recycling of space debris.

\section{Figure 3}

A proposed system design for the collection and recycling of space debris. 


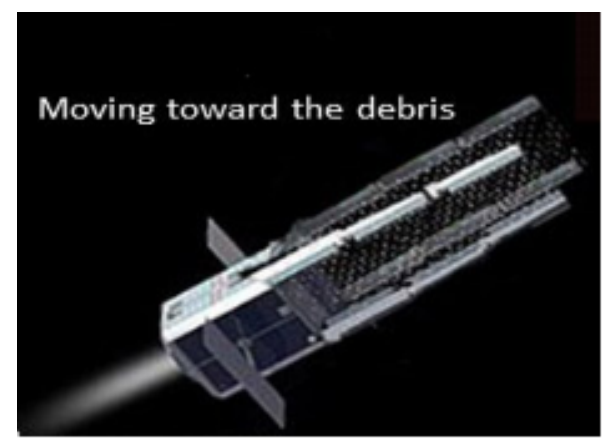

Figure 4: Stage 1

\section{Figure 4}

\section{Stage 1}

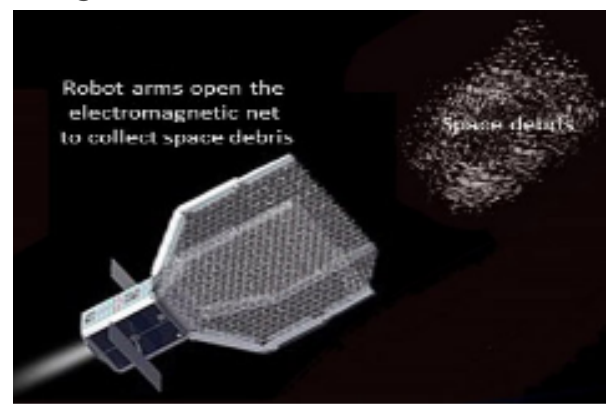

Figure 5: Stage 2

\section{Figure 5}

Stage 2

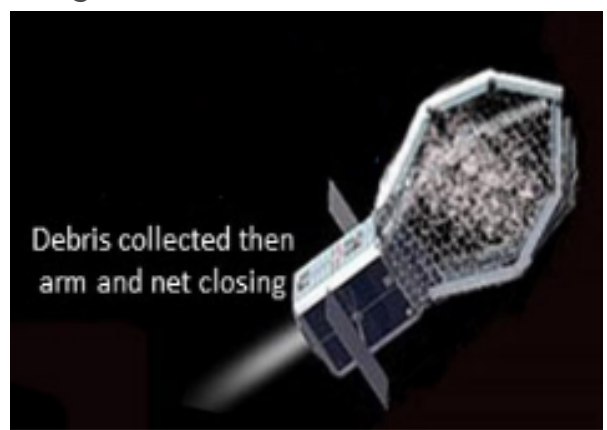

Figure 6: Stage3

Figure 6

Stage 3 


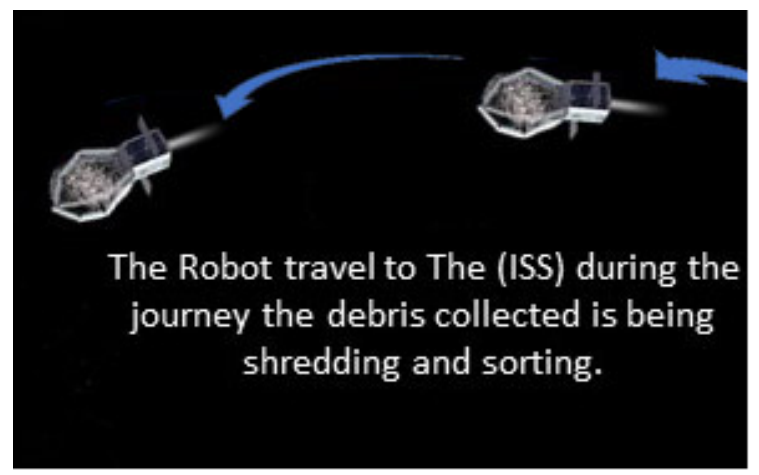

Figure 7: Stage 4

Figure 7

Stage 4

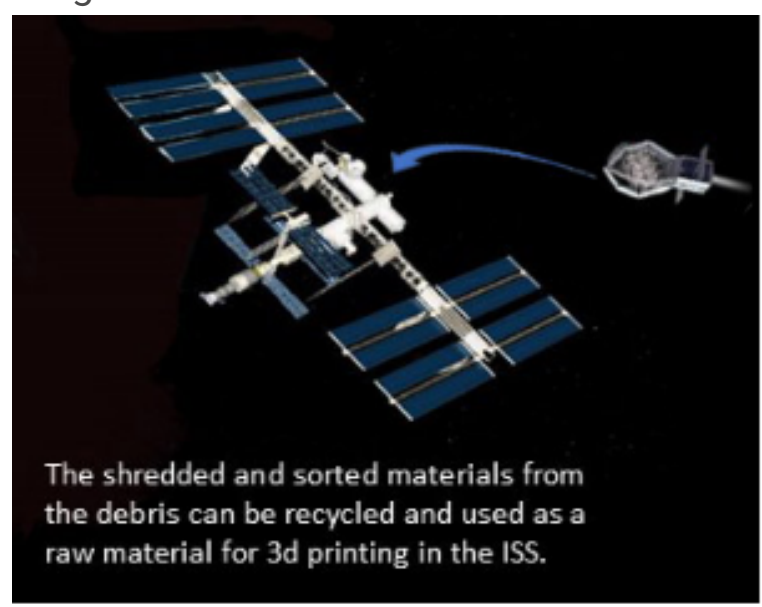

Figure 8: Stage 5

Figure 8

Stage 5 